REVISTA MATEMATICA de la

Universidad Complutense de Madrid

Volumen 7, número 1: 1994

http://dx.doi.org/10.5209/rev_REMA.1994.v7.n1.17790

\title{
Link Homotopy Invariants of Graphs in $R^{3}$
}

\author{
KOUKI TANIYAMA
}

ABSTRACT. In this paper we define a link homotopy invariant of spatial graphs based on the second degree coefficient of the Conway polynomial of a knot.

\section{INTRODUCTION}

Throughout this paper we work in the piccewise linear category. Let $G$ be a finite graph without loops and multiple edges. Then there are various embeddings of $G$ into the three-dimensional Euclidean space $R^{3}$. Two embeddings $f, g: G \rightarrow R^{3}$ are said to be link homotopic if $g$ is

1991 Mathematics Subject Classification: 57M25, 57M15, $05 \mathrm{C} 10$

Editorial Complutense. Madrid, 1994. 
obtained from $f$ by a finite sequence of self-crossing changes (Fig. 1.1) and ambient isotopy.
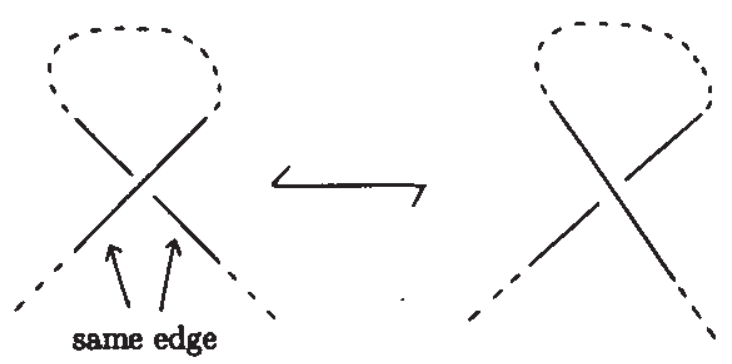

Fig. 1.1

Two edges of $G$ are called adjacent if they have a vertex in common. Two embeddings $f, g: G \rightarrow R^{3}$ are called weakly link homotopic if $g$ is obtained from $f$ by a finite sequence of crossing changes of adjacent edges (Fig. 1.2) and ambient isotopy.
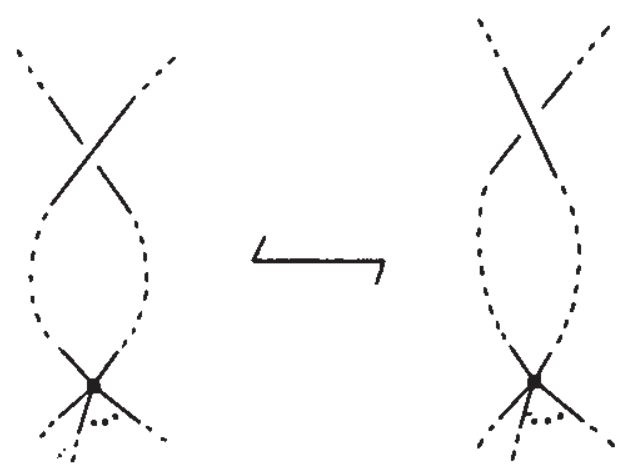

Fig. 1.2 
We note that a self-crossing change is replaced by crossing changes of adjacent edges as illustrated in Fig. 1.3. Therefore link homotopy implies weak link homotopy.
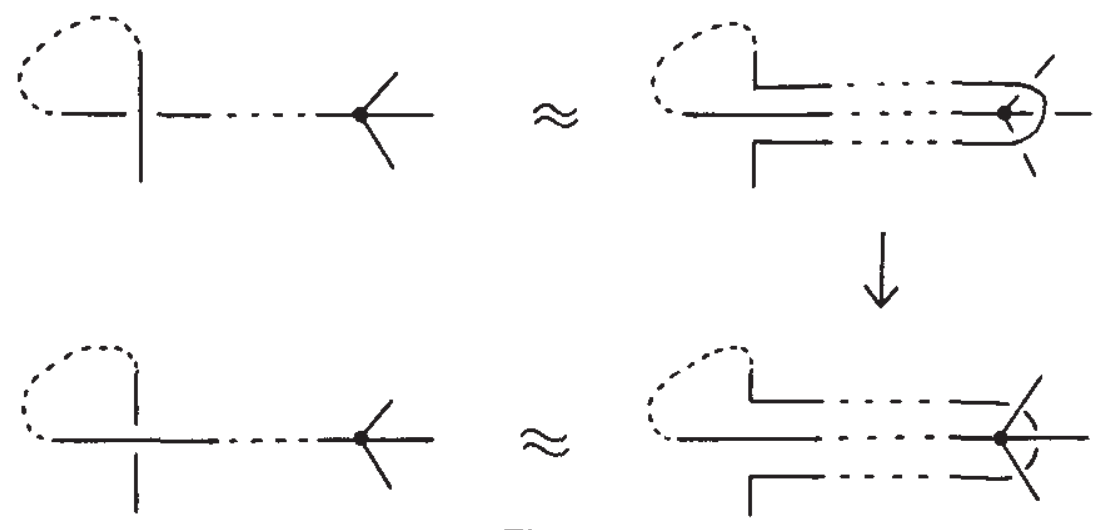

Fig. 1.3

An $n$-cycle is a graph with $n$ vertices that is homeomorphic to a circle. When $G$ is a disjoint union of cycles our link homotopy and weak link homotopy coincide with Milnor's link homotopy defined in [4].

The purpose of this paper is to define link homotopy invariants and weak link homotopy invariants for an arbitrary graph $G$. By the fundamental theorem in [7] we have that a link homotopy invariant is an $I$-equivalence invariant and hence an isotopy invariant and also a cobordism invariant. Conversely a homology invariant is a link homotopy invariant. Thus Wu's invariant (see [8]) is a weak link homotopy invariant and hence a link homotopy invariant. Except the case that $G$ is a disjoint union of cycles, the author knows no other link homotopy invariants and weak link homotopy invariants.

A cycle of a graph $G$ is a subgraph of $G$ that is a cycle. Let $\Gamma=$ $\Gamma(G)$ be the set of all cycles of $G$. Let $Z$ be the integers. Let $n$ be a non-negative integer. Let $Z_{n}=\{0,1,2, \ldots, n-1\}$ if $n>0$. Let $Z_{0}=Z$. Let $\omega: \Gamma \rightarrow Z_{n}$ be a map. We call $\omega$ a weight on $\Gamma$. For an embedding $f: G \rightarrow R^{3}$ we define $\alpha_{\omega}(f) \in Z_{n}$ by

$$
\alpha_{\omega}(f) \equiv \sum_{\gamma \in \Gamma} \omega(\gamma) a_{2}(f(\gamma))(\bmod n)
$$


where $a_{2}(K)$ is the coefficient of $z^{2}$ in the Conway polynomial $\nabla_{K}(z)$ of a knot $K$. We will show that if a weight $\omega$ satisfies certain conditions then $\alpha_{\omega}$ is a (weak) link homotopy invariant.

We remark here that the modulo 2 reduction of $a_{2}(K)$ equals the Arf invariant of $K[3]$. Therefore when $G$ is the complete graph $K_{7}, n=2$ and

$$
\omega(\gamma)= \begin{cases}1 & \text { if } \gamma \text { is a } 7-\text { cycle } \\ 0 & \text { otherwise }\end{cases}
$$

$\alpha_{\omega}$ equals an invariant defined in [2]. In [2] Gordon proved that $\alpha_{\omega}$ is invariant under any crossing change. He found a particular embedding $f: K_{7} \rightarrow R^{3}$ such that $\alpha_{\omega}(f) \equiv 1(\bmod 2)$. Therefore $\alpha_{\omega}(g) \equiv 1(\bmod$ 2) for any embedding $g: K_{7} \rightarrow R^{3}$. Since $a_{2}$ (unknot) $=0$ he could conclude that every spatial embedding of $K_{7}$ contains a nontrivially knotted 7-cycle.

For our purpose it is enough that $\alpha_{\omega}$ is invariant under a selfcrossing change or a crossing change of adjacent edges. In this sense the idea in [2] was a great hint of this paper. We also remark here that our definition of $\alpha_{\omega}(f)$ generalizes Shimabara's generalization of Gordon's invariant [6].

Let $e$ be an edge of $G$. We give an arbitrary orientation to $e$. Let $\Gamma_{e}$ be a subset of $\Gamma$ defined by

$$
\Gamma_{e}=\{\gamma \in \Gamma \mid \gamma \supset e\}
$$

We give an orientation to each $\gamma \in \Gamma_{e}$ by the orientation of $e$. We say that a weight $\omega: \Gamma \rightarrow Z_{n}$ is balanced on $e$ if the homological sum $\Sigma_{\gamma \in \Gamma_{e}} \omega(\gamma) \gamma$ is zero in $H_{1}\left(G ; Z_{n}\right)$. We remark that this property does not depend on the choice of the orientation of $e$.

Lemma 1.1. Let $\omega: \Gamma(G) \rightarrow Z_{n}$ be a weight that is balanced on an edge e of $G$. If an embedding $g: G \rightarrow R^{3}$ is obtained from an embedding $f: G \rightarrow R^{3}$ by a self-crossing change of the edge e then

$$
\alpha_{\omega}(f) \equiv \alpha_{\omega}(g) \quad(\bmod n)
$$

As an immediate corollary we have: 
Theorem 1.2. Let $\omega: \Gamma(G) \rightarrow Z_{n}$ be a weight that is balanced on each edge of $G$. Then $\alpha_{\omega}$ is a link homotopy invariant. Namely if two embeddings $f, g: G \rightarrow R^{3}$ are link homotopic then

$$
\alpha_{\omega}(f) \equiv \alpha_{\omega}(g) \quad(\bmod n)
$$

Let $e_{1}$ and $\epsilon_{2}$ be adjacent edges of $G$. We give an arbitrary orientation to $e_{1}$. Let $\Gamma_{e_{1}, e_{2}}$ be a subset of $\Gamma$ defined by

$$
\Gamma_{e_{1}, e_{2}}=\left\{\gamma \in \Gamma \mid \gamma \supset e_{1}, e_{2}\right\} .
$$

We give an orientation to each $\gamma \in \Gamma_{e_{1}, e_{2}}$ by the orientation of $e_{1}$. We say that a weight $\omega: \Gamma \rightarrow Z_{n}$ is balanced on a pair of adjacent edges $\left(e_{1}, e_{2}\right)$ if the homological sum $\Sigma_{\gamma \in \Gamma_{e_{1}, e_{2}}} \omega(\gamma) \gamma$ is zero in $H_{1}\left(G ; Z_{n}\right)$.

Lemma 1.3. Let $\omega: \Gamma(G) \rightarrow Z_{n}$ be a weight that is balanced on a pair of adjacent edges $\left(e_{1}, e_{2}\right)$ of $G$. If an embedding $g: G \rightarrow R^{3}$ is obtained from an embedding $f: G \rightarrow R^{3}$ by a crossing change between $e_{1}$ and $e_{2}$ then

$$
\alpha_{\omega}(f) \equiv \alpha_{\omega}(g) \quad(\bmod n) .
$$

As an immediate corollary we have:

Theorem 1.4. Let $\omega: \Gamma(G) \rightarrow Z_{n}$ be a weight that is balanced on each pair of adjacent edges of $G$. Then $\alpha_{\omega}$ is a weak link homotopy invariant. Namely if two embeddings $f, g: G \rightarrow R^{3}$ are weakly link homotopic then

$$
\alpha_{\omega}(f) \equiv \alpha_{\omega}(g) \quad(\bmod n) .
$$

This paper is organized as follows. In $\S 2$ we prove Lemma 1.1 and Lenma 1.3. In $\S 3$ we show some examples. In $\$ 4$ we show that Milnor's $\mu$-invariant for 3-component homologically unlinked links can be re-defined via a weak link homotopy invariant of a certain graph.

\section{PROOFS OF LEMMA 1.1 AND LEMMA 1.3}

Proof of Lemma 1.1. We recall the equality

$$
\text { (*) } a_{2}\left(K_{+}\right)-a_{2}\left(K_{-}\right)=\ell k\left(L_{0}\right)
$$


where $K_{+}, K_{-}$and $L_{0}$ are knots and a two-component link as illustrated in Fig. 2.1 and $\ell k$ denotes the linking number [3].<smiles>C1CCC2(CC1)CCCC2</smiles>

$K_{+}$<smiles>[Y]1CCCCC12CCCC2</smiles>

K.<smiles>C1CCCCCC1</smiles>

$L_{0}$

Fig. 2.1

Let $\gamma$ be a cycle in $\Gamma_{e}$. We recall that $\gamma$ is oriented by the orientation of $e$. We may suppose without loss of generally that $f(\gamma)$ and $g(\gamma)$ are related as illustrated in Fig. 2.2 (a) and (b). Let $L_{f, g}(\gamma)=\ell_{f, g}(\gamma) \cup$ $m_{f, g}(\gamma)$ be the 2-component link as illutrated in Fig. 2.2 (c).

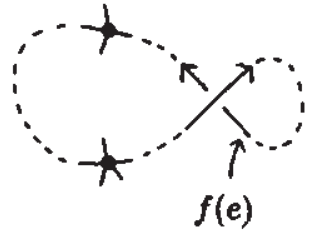

$f(\gamma)$

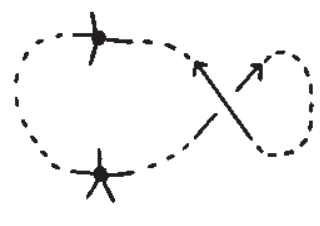

$g(\gamma)$

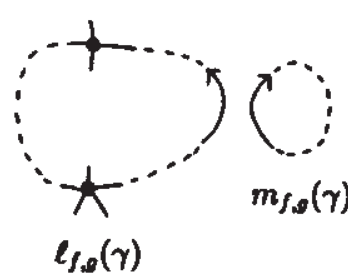

$L_{\rho g}(\gamma)$

(a)

(b)

(c) 
Then we have

$$
\begin{gathered}
\alpha_{\omega}(f)-\alpha_{\omega}(g) \equiv \sum_{\gamma \in \Gamma} \omega(\gamma) a_{2}(f(\gamma))-\sum_{\gamma \in \Gamma} \omega(\gamma) a_{2}(g(\gamma)) \\
\equiv \sum_{\gamma \in \Gamma} \omega(\gamma)\left(a_{2}(f(\gamma))-a_{2}(g(\gamma))\right) \equiv \sum_{\gamma \in \Gamma_{e}} \omega(\gamma)\left(a_{2}(f(\gamma))-a_{2}(g(\gamma))\right) \\
\equiv \sum_{\gamma \in \Gamma_{e}} \omega(\gamma) \ell k\left(\ell_{f, g}(\gamma), m_{f, g}(\gamma)\right)(\bmod n) .
\end{gathered}
$$

Since $m_{f, g}(\gamma)=m_{f, g}\left(\gamma^{\prime}\right)$ for any $\gamma, \gamma^{\prime} \in \Gamma_{e}$ we may write $m_{f, g}(\gamma)$ as $m_{f, g}$. Since linking number is a homological invariant we have

$$
\begin{gathered}
\sum_{\gamma \in \Gamma_{e}} \omega(\gamma) \ell k\left(\ell_{f, g}(\gamma), m_{f, g}\right) \equiv \sum_{\gamma \in \Gamma_{e}} \ell k\left(\omega(\gamma) \ell_{f, g}(\gamma), m_{f, g}\right) \\
\equiv \ell k\left(\sum_{\gamma \in \Gamma_{e}} \omega(\gamma) \ell_{f, g}(\gamma), m_{f, g}\right) \quad(\bmod n) .
\end{gathered}
$$

Since $\omega$ is balanced on $e$ we have that the homological sum

$$
\sum_{\gamma \in \Gamma_{e}} \omega(\gamma) \ell_{f, g}(\gamma) \equiv 0 \quad(\bmod n)
$$

Therefore we have

$$
\ell k\left(\sum_{\gamma \in \Gamma_{e}} \omega(\gamma) \ell_{f, g}(\gamma), m_{f, g}\right) \equiv \ell k\left(0, m_{f, g}\right) \equiv 0 \quad(\bmod n)
$$

This completes the proof.

Proof of Lemma 1.3. The proof is similar to that of Lemma 1.1. We note that one of the two components of the smoothed link is common 
for all $\gamma \in \Gamma_{e_{1}, e_{2}}$ as in the case of Lemma 1.1, see Fig. 2.3.
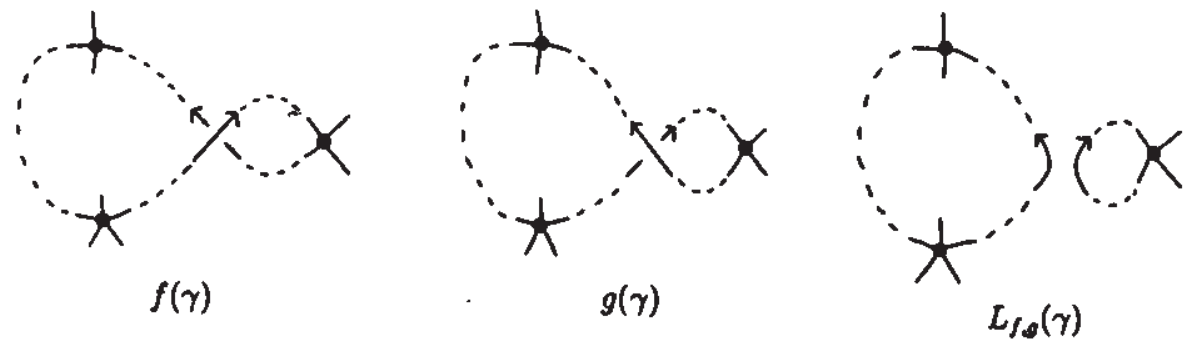

Fig. 2.3

Therefore the same proof works.

\section{EXAMPLES}

Example 3.1. Let $G$ be the complete graph $K_{4}$. Let $n=0$ and let $\omega: \Gamma\left(K_{4}\right) \rightarrow Z$ be a weight defined by

$$
\omega(\gamma)= \begin{cases}1 & \text { if } \gamma \text { is a } 4 \text {-cycle } \\ -1 & \text { if } \gamma \text { is a } 3 \text {-cycle }\end{cases}
$$

Then it is easily checked that $\omega$ is balanced on each edge of $K_{4}$. Therefore $\alpha_{\omega}$ is a link homotopy invariant. 
Let $j$ be an integer and let $f_{j}: K_{4} \rightarrow R^{3}$ be an embedding illustrated by Fig. 3.1 where the box denotes $2 j-1$ right-handed half twists when $j>0,-2 j+1$ left-handed half twists when $j \leq 0$.

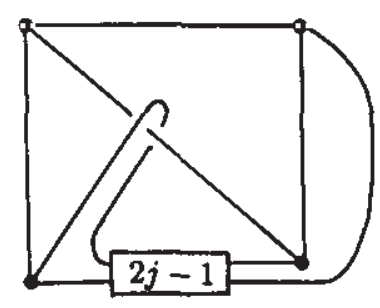

$f_{j}\left(K_{4}\right)$

Fig. 3.1

Then $f_{j}\left(K_{4}\right)$ contains at most one nontrivial knot. The knot is a twisted knot. Since a twisted knot has unknotting number one $a_{2}$ is easily calculated by the equality (*). Then we have $\alpha_{\omega}\left(f_{j}\right)=j$.

Example 3.2. Let $G=K_{5}, n=0$ and $\omega: \Gamma\left(K_{5}\right) \rightarrow Z$ a weight defined by

$$
\omega(\gamma)= \begin{cases}1 & \text { if } \gamma \text { is a } 5 \text {-cycle } \\ -1 & \text { if } \gamma \text { is a } 4 \text {-cycle } \\ 0 & \text { if } \gamma \text { is a } 3 \text {-cycle }\end{cases}
$$

Then it is easily checked that $\omega$ is balanced on each pair of adjacent edges of $K_{5}$. Thus $\alpha_{\omega}$ is a weak link homotopy invariant. 
Let $j$ be an integer. Let $f_{j}: K_{5} \rightarrow R^{3}$ be an embedding illustrated by Fig. 3.2 .

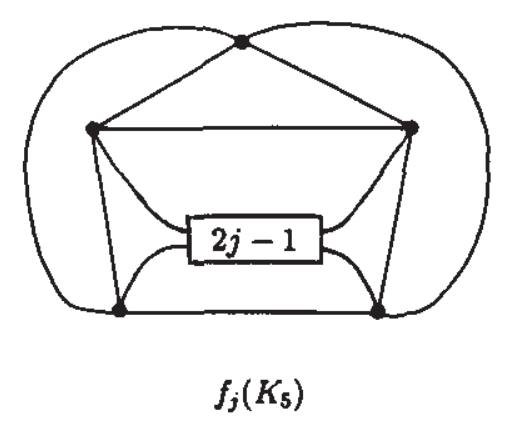

Fig. 3.2

Then at most two 5-cycles and a 4-cycle can be nontrivial knots. They are all the $(2,2 j-1)$-torus knot. From the equality (*) we have that $a_{2}((2,2 j-1)$-torus knot $)=\frac{j(j-1)}{2}$. Therefore we have that $\alpha_{\omega}\left(f_{j}\right)=$ $\frac{j(j-1)}{2}$.

It is known in [8] that $\left\{f_{j} \mid j \in Z\right\}$ is a complete list of the homology classes of embeddings of $K_{5}$ into $R^{3}$. In [5] we will show that homology implies weak link homotopy when $G=K_{5}$. Therefore $\left\{f_{j} \mid j \in Z\right\}$ is also a complete list of weak link homotopy classes. Thus $\alpha_{\omega}$ classifies the embeddings of $K_{5}$ into $R^{3}$ up to weak link homotopy and mirtor image. 


\section{3-COMPONENT HOMOLOGICALLY UNLINKED}

\section{LINKS}

Let $G$ be the graph of Fig. 4.1.

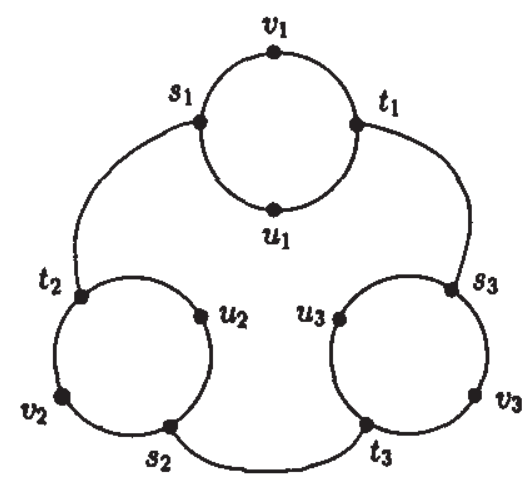

Fig. 4.1

Let $n=0$ and let $\omega: \Gamma(G) \rightarrow Z$ be a weight defined by

$$
\omega(\gamma)=\left\{\begin{array}{cc}
1 & \text { if } \gamma \text { is a } 9 \text {-cycle that contains zero or two of } \\
v_{1}, v_{2} \text { and } v_{3} \\
-1 & \text { if } \gamma \text { is a } 9 \text {-cycle that contains one or three of } \\
v_{1}, v_{2} \text { and } v_{3} \\
0 & \text { if } \gamma \text { is a } 4-\text { cycle. }
\end{array}\right.
$$

Then $\omega$ is balanced on each pair of adjacent edges of $G$. Thus $\alpha_{\omega}$ is a weak link homotopy invariant.

A 3-component ordered oriented link $L=\ell_{1} \cup \ell_{2} \cup \ell_{3}$ is called homologically unlinked if $\ell k\left(\ell_{1}, \ell_{2}\right)=\ell k\left(\ell_{2}, \ell_{3}\right)=\ell k\left(\ell_{3}, \ell_{1}\right)=0$. Let $H$ be the subgraph of $G$ that is the disjoint union of three 4-cycles of 
$G$. Let $f: H \rightarrow R^{3}$ be an embedding. Let $\ell_{i}(f)=f\left(v_{i} s_{i} u_{i} t_{i} v_{i}\right)(i=$ $1,2,3)$. Then $L(f)=\ell_{1}(f) \cup \ell_{2}(f) \cup \ell_{3}(f)$ is a 3 -component ordered oriented link.

Theorem 4.1. Let $f, g: G \rightarrow R^{3}$ be embeddings such that both $L\left(\left.f\right|_{H}\right)$ and $L\left(\left.g\right|_{H}\right)$ are homologically unlinked. Then $f$ and $g$ are weakly link homotopic if and only if $\left.f\right|_{H}$ and $\left.g\right|_{H}$ are weakly link homotopic.

Proof. The 'only if' part is clear. We show 'if' part. Suppose that $\left.f\right|_{H}$ is weakly link homotopic to $\left.g\right|_{H}$. Then $f$ is weakly link homotopic to an embedding, still denoted by $f$, so that $\left.f\right|_{H}=\left.g\right|_{H}$. It is sufficient to show that a crossing change between the edge $s_{i} t_{i+1}$ and an edge of $G$ is realized by a weak link homotopy (here we consider the suffix modulo 3). By replacing a crossing change by some crossing changes as in Fig. 1.3 we have that a crossing change between $s_{i} t_{i+1}$ and an edge that is not on the cycle $v_{i+2} s_{i+2} u_{i+2} t_{i+2} v_{i+2}$ is realized by some crossing changes of adjacent edges. Then by the symmetry of $G$ is sufficient to show that a crossing between $s_{1} t_{2}$ and $v_{3} s_{3}$ is realized by a weak link homotopy. We choose a small ball $B^{3}$ near the crossing where the crossing change is desired, see Fig. 4.2.

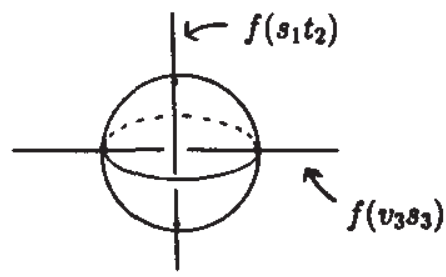

Fig. 4.2

Step 1. By a weak link homotopy outside of $B^{3}$ we deform $f$ so that $\ell_{1}(f) \cup \ell_{2}(f)$ is a trivial 2-component link.

Step 2. We choose a disk $D^{2}$ in general position so that $\partial D^{2}=$ $\ell_{1}(f), D^{2} \cap \ell_{2}(f)=\emptyset$ and $D^{2} \cap B^{3}=\emptyset$. 
Step 3. We remove the intersection if any of $D^{2}$ and $f\left(s_{1}, t_{2}\right)$ by a weak link homotopy outside of $B^{3}$.

Step 4. We perform the crossing change by a weak link homotopy as illustrated in Fig. 4.3.
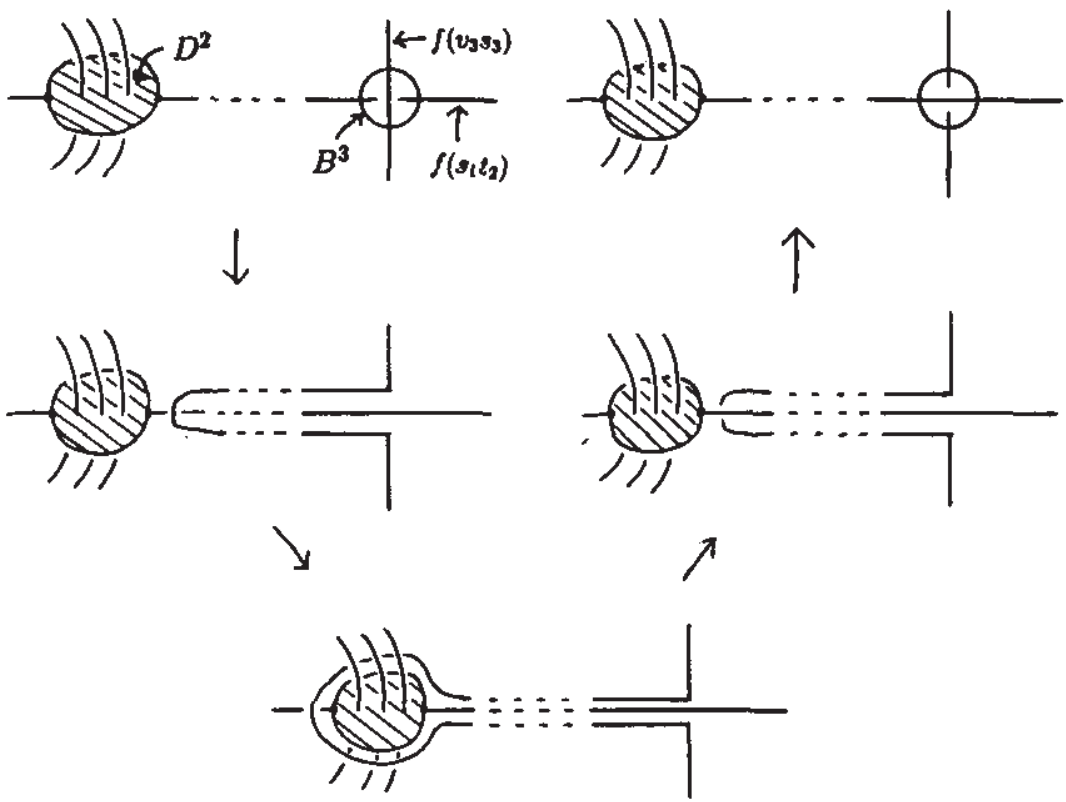

Fig. 4.3

Step 5. We re-fix the 3-ball $B^{3}$ and retrace one's steps from Step 3 to Step 1.

Thus we have the desired crossing change.

Let $L$ be a homologically unlinked 3-component ordered oriented link. Let $f: G \rightarrow R^{3}$ be an embedding such that $L\left(\left.f\right|_{H}\right)=L$. Then by Theorem $4.1 \alpha_{\omega}(f)$ is a well-defined weak link homotopy invariant of $L$. Since weak link homotopy equals link homotopy for links $\alpha_{\omega}(f)$ 
is a link homotopy invariant of $L$. It is known in [4] that 3-component homologically unlinked links are classified up to link homotopy by Milnor's $\mu$-invariant. Let $j$ be an integer and let $L_{j}$ be a link illustrated in Fig. 4.4.

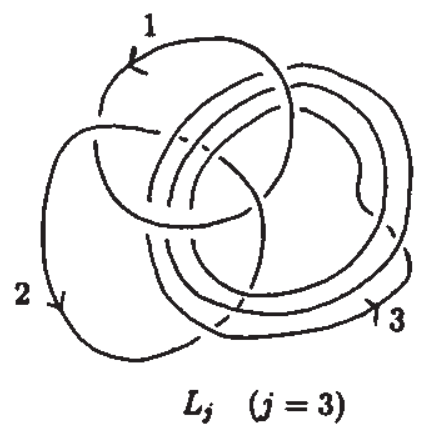

Fig. 4.4

Then $\mu\left(L_{j}\right)=j$ and $\left\{L_{j} \mid j \in Z\right\}$ is the complete list of link homotopy classes [4]. Let $f_{j}: G \rightarrow R^{3}$ be an embedding illustrated in 
Fig. 4.5.

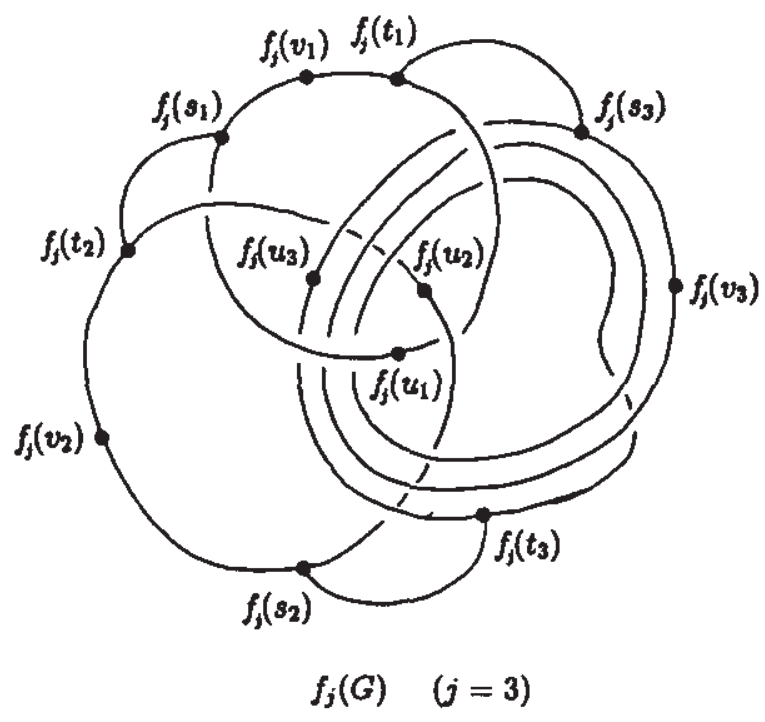

Fig. 4.5

Then $L\left(f_{j}\right)=L_{j}$. It is easy to check that $f_{j}(G)$ contains at most two nontrivial knots that are twisted knots. Then we have $\alpha_{\omega}\left(f_{j}\right)=j$. Thus Milnor's $\mu$-invariant is re-defined, cf. [1].

Acknowledgements. The author would like to thank Professor Shin'ichi Suzuki for his encouragement.

\section{References.}

[1] Cochran, T.D. Concordance invariance of coefficients of Conway's link polynomial. Invent. Math. 82, (1985), 527-541.

[2] Conway, J.H. and McA. Gordon, C. Knots and links in spatial graphs. J. Graph Theory, 7, (1983), 445-453. 
[3] Kauffman, L. On knots. Ann. of Math. Studies, 115, Princeton Univ. Press, Princeton, N.J., (1987).

[4] Milnor, J. Link groups. Ann. Math., 59, (1954), 177-195.

[5] Motohashi, T. and Taniyama, K. Homology and weak link homotopy of graphs in $R^{3}$, in preparation.

[6] Shimabara, M. Knots in certain spatial graphs, Tokyo J. Math., 11, (1988), 405-413.

[7] Taniyama, K. Cobordism, homotopy and homology of graphs in $R^{3}$, to appear in Topology.

[8] Taniyama, K. Homology classification of spatial embeddings of a graph, preprint. 